\title{
Left Atrial Appendage Closure for Stroke Prevention
}

Evan Kimber

Editorial Assistant

Citation: EMJ Int Cardiol. 2021;9[1]:22-24.

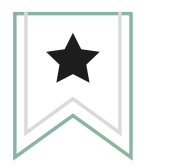

\begin{abstract}
MPLEMENTING an effective patient-selection approach, integrating device choice, procedural planning, and patient follow-up, have proven crucial to the success of left atrial appendage closure (LAAC) therapy for atrial fibrillation (AF). This was the focus of the EuroPCR 2021 'Structural Theatre' session, which compared LAAC and direct oral anticoagulant (DOAC) treatment avenues for stroke prevention.
\end{abstract}

In this session, Patrick Calvert, from Royal Papworth Hospital in Cambridge, UK, gave a presentation on patient selection, alongside Ole De Backer of Copenhagen University Hospital, Denmark, who discussed device selection and planning using CT imagery. Ivan Kuntjoro, from the National University Heart Centre of Singapore, briefly outlined left atria morphology, and Farell Hellig of Sunninghill Hospital, Johannesburg, South Africa, introduced procedural plans in a step-by-step approach while performing a live procedure. The session was concluded by Edgar Tay, UK Royal College of Physicians, who gave his insights on several of these topics and concluded the stream with how to optimise patient follow-up care.

\section{HOW TO SELECT SUITABLE PATIENTS FOR LEFT ATRIAL APPENDAGE CLOSURE}

Calvert started his presentation by clarifying that LAAC reduces embolic stroke risk in the absence of oral anticoagulation (OAC) therapy. Calvert highlighted DOAC as the first-choice treatment for AF patients and listed the criteria for selecting $L A A C$, emphasising that considering a balance of risks is crucial when weighing up these therapy options. He highlighted the importance of consulting a multidisciplinary team of professionals in order to incorporate a clear, open discussion with the patient about preferences and risks. In essence, LAAC is most useful to patients who cannot tolerate DOAC and want to have the procedure, but should not be used in cases with rheumatic heart disease (RHD), Calvert emphasised.

The criteria for use of LAAC include clear evidence from ECG of documented AF, and an absence of mitral stenosis (MS), as well as strokerisk scores less than 3 and 2 using $\mathrm{CHA}_{2} \mathrm{DS}_{2}$-VASC and HAS-BLED models, respectively. The invasive and complex nature of the procedure, with the possibility of no tangible symptomatic benefits, underpins risks of the procedure; however, when patients on DOAC continue to have embolic strokes, LAAC intervention is necessary. Considerations should include the robustness of a patient and the possibility of major complications during surgery. 
"The invasive and complex nature of the procedure, with the possibility of no tangible symptomatic benefits, underpins risks of the procedure; however, when patients on DOAC continue to have embolic strokes, LAAC intervention is necessary"

Expanding upon these parameters, Tay stated data have shown that patients receiving warfarin can effectively be treated with LAAC and agreed there is value in an open patient-multidisciplinary team discussion.

\section{DEVELOPING AN APPROACH WITH APPROPRIATE DEVICE SELECTION}

De Backer outlined the key points for appropriate device selection and procedural planning, recommending CT-based sizing methods ahead of transoesophageal echocardiography (TEE). De Backer stated, "the accuracy of LAA sizing cannot be over-emphasised," and that "optimised pre-procedural planning for percutaneous LAA closure based on CT analysis is the gold standard.".

Building upon this, Guiseppe Tarantini from the University of Padua Medical School, Italy, commented: "One device cannot fit all LAAs". However, he added, "one device can fit more than one LAA morphology."

\section{ADVANCEMENTS IN PROCEDURAL PLANNING}

Tay complimented the shift towards CT imaging and its role in aiding a step-by-step approach. He suggested planning is swifter and more accurate when conducted in this fashion. To present this, Hellig described the case of a patient

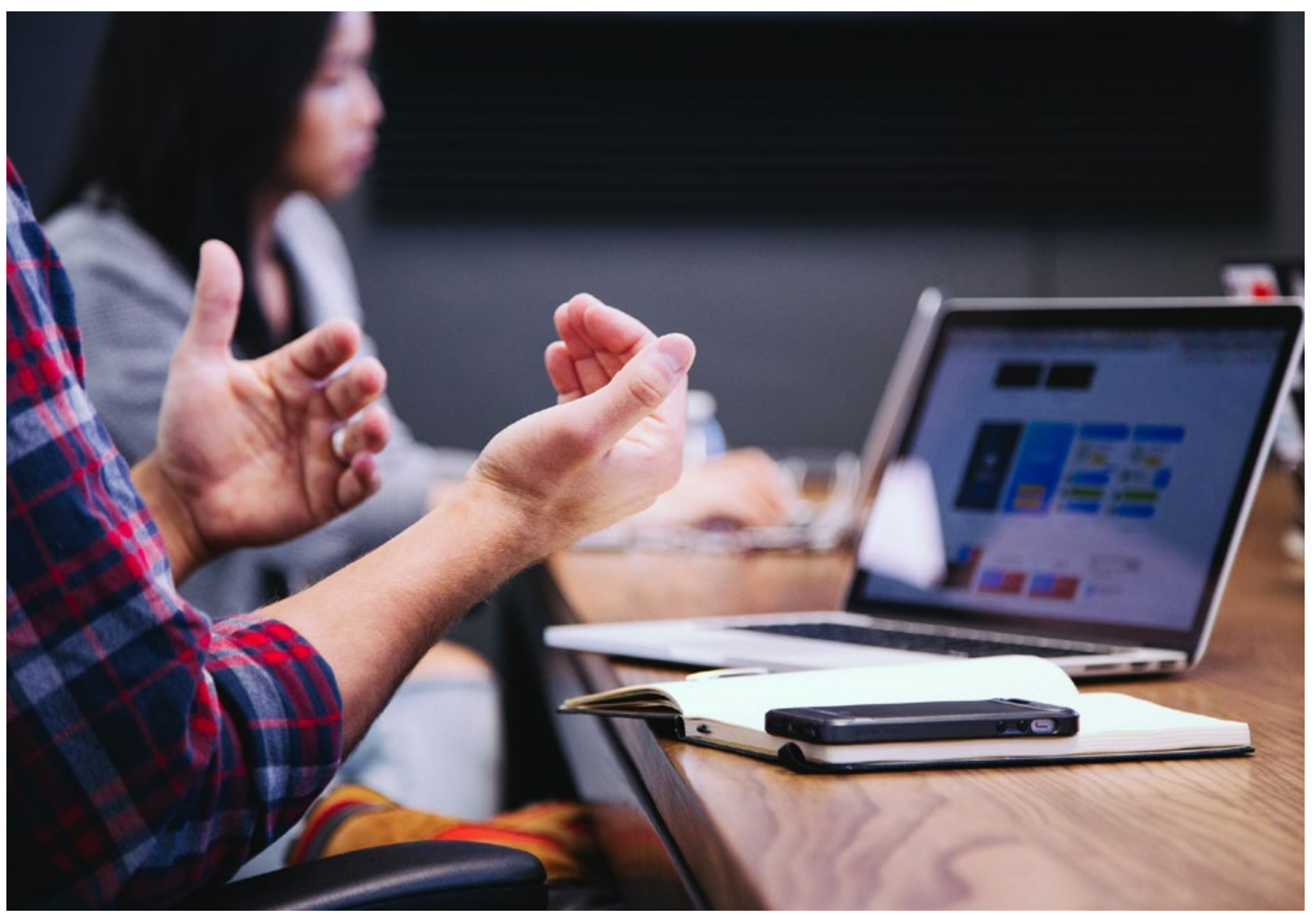




\section{"optimised pre-procedural \\ planning for percutaneous \\ LAA closure based on

$$
\begin{aligned}
& \text { CT analysis is the } \\
& \text { gold standard." }
\end{aligned}
$$

with paroxysmal $A F$ and gastrointestinal tract bleeding where DOAC was halted. De Backer gave an example of how CT software can be used in this scenario to visually aid the decisionmaking process for selecting either a 'Watchman FLX' or 'Amulet' device. He stressed how "crucial" it is that cardiologists do the pre-procedure CT analysis themselves.

The value of this approach was observed in the live case from Sunninghill Hospital, Sandton, South Africa, which gave a demonstration of a complete seal in a complex situation. Pre-surgical analysis paid off when the device selected was repositioned multiple times successfully. Hellig alluded to the importance of selecting a device using both pre- and intra-procedural guidelines and confirming a stable position and seal before release.

\section{OPTIMISING POST-PROCEDURE CARE}

Last on the agenda was optimising postprocedure follow-up and care, and Tay stated, "the choice of post-procedure care is dependent on who the procedure is on." He explained that OAC should be used for the first 45 days if it can be tolerated, TEE should then be employed to check for device-related thrombosis, and if clear the patient should then be de-escalated to antiplatelet therapy for 6 months. Tay confirmed there will be complications and 1 month of dual antiplatelet therapy followed by prescription of aspirin for those that cannot use OAC. In this way, post-procedural medication and follow-up is tailored according to a patient's clinical picture.

\section{FUTURE IMPLICATIONS}

Looking ahead, the conversations in this EuroPCR event will help cardiologists to take personal responsibility when using $\mathrm{CT}$ imaging and to adopt a more open and diverse approach towards AF treatment.

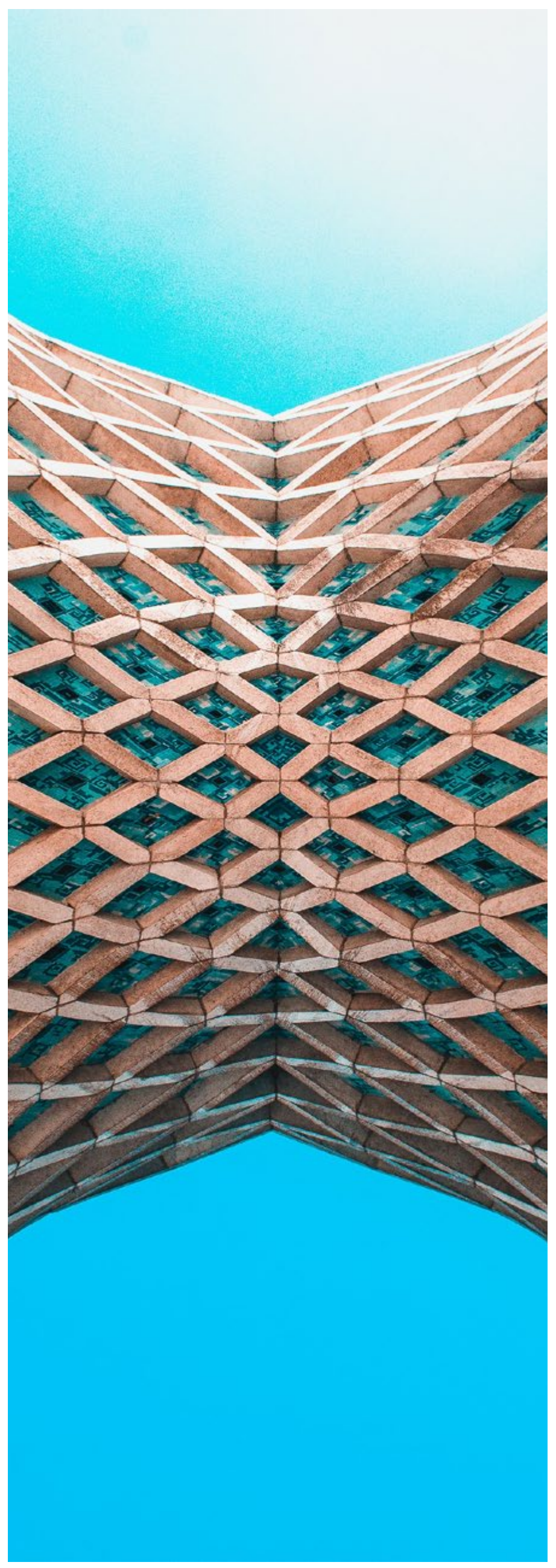

